\title{
¿Piedra, papel o tijeras? La imprenta y Spotify como innovaciones tecnológicas
}

\author{
Leonardo A. González
}

\section{Resumen}

Este artículo es una reflexión sobre las coyunturas culturales provocadas por innovaciones tecnológicas como la imprenta, en el siglo xvı, y la aplicación digital Spotify, en el presente siglo xxı. Ambos casos se explican desde una mirada crítica a través de tres interrogantes: si realmente la imprenta fue un parteaguas, cómo es que los libros sembraron el pensamiento moderno, y si hay alguna situación presente que puede compararse con el fenómeno de los libros impresos.

Para responder a estas preguntas, el texto se construye como un argumento: dos premisas y una conclusión. Cabe mencionar que estas consideraciones son producto de una serie de reflexiones desde la práctica docente, dentro del área de Ciencias Sociales, así como, una respuesta a las preocupaciones sobre el futuro de la educación.

Palabras clave: libro, imprenta, innovación tecnológica, coyuntura cultural, universo digital, Spotify.

\section{ROCK, PAPER OR SCISSORS? THE PRINTING PRESS AND SPOTIFY AS TECHNOLOGICAL INNOVATIONS}

\begin{abstract}
This article is a critical reflection about the cultural conjunctures caused by technological innovations such as printing press, in the Sixteenth Century, and the digital application Spotify, in the present. Both cases are explained from a critical view through three questions: Was the printing press a watershed? How do books sow modern thinking? Is there any present situation that can be compared to the phenomenon of printed books?

To answer these questions, this paper is constructed as an argument, with two premises and a conclusion. Worth mentioning is the fact that these considerations are the product of teaching practice within the area of Social Sciences and a response to the concerns about the future of education.
\end{abstract}

Keywords: book, printing press, technological innovations, cultural conjunctures, digital universe, Spotify. 


\section{Leonardo A. González}

Es profesor de asignatura en la Academia de Ciencias Sociales e Historia, del Colegio de Bachilleres de la Ciudad de México. Realizó sus estudios profesionales y de posgrado en la unAm, en el área de Humanidades. Destaca su amplia formación en la investigación histórica en temas como la cartografía indígena y colonial, y en la docencia de Historia Universal y de México. Actualmente está incubando un emprendimiento educativo de base tecnológica y con impacto social, en la Coordinación de Innovación y Desarrollo de la unam. Cabe destacar que posee un gran interés por la historia de la ciencia y la tecnología, así como por la literatura y la filmografía medieval. 


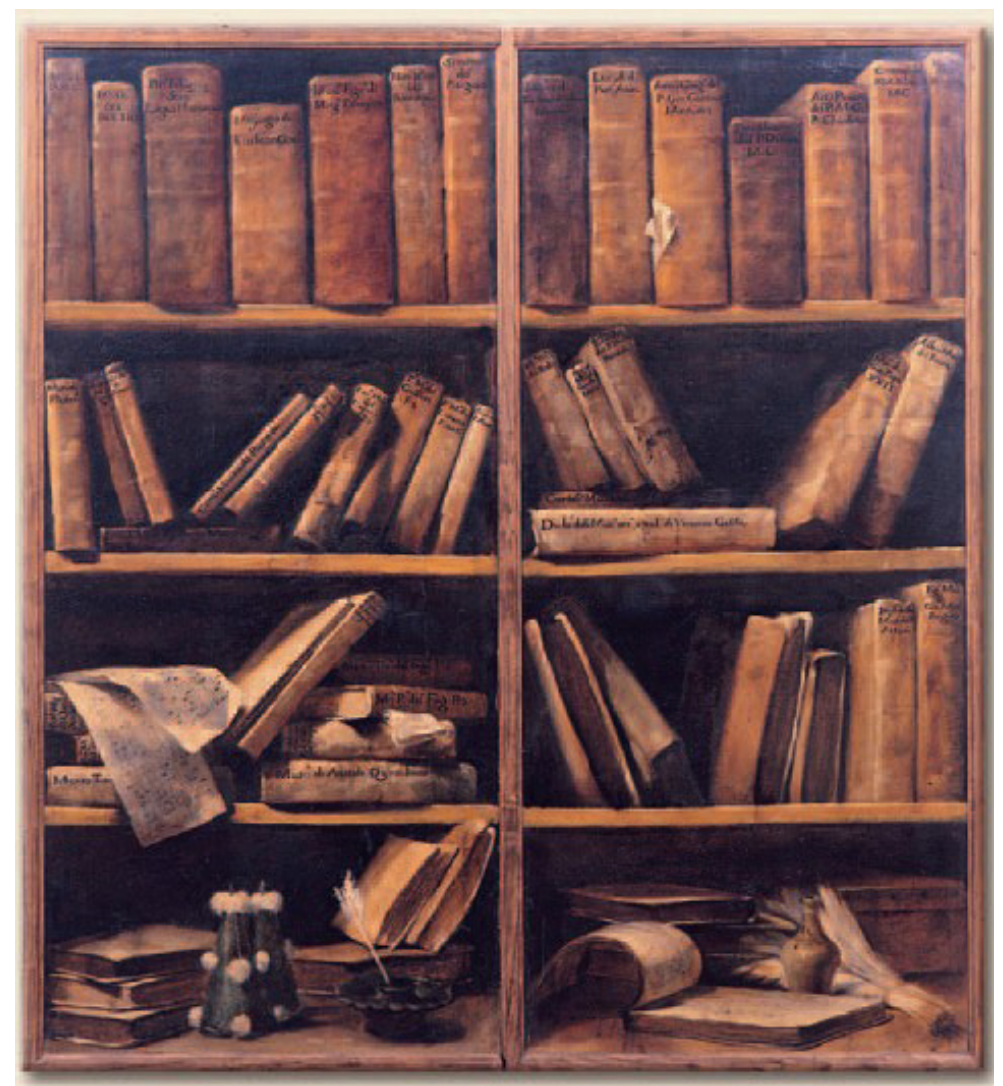

\section{Introducción}

En la Modernidad, la mayor parte de los procesos de enseñanza tienen como base el conocimiento escrito, es decir, los libros. Se trata de una herencia del cristianismo medieval y si rascamos más en la historia podemos encontrar tradiciones aún más antiguas.

En la escuela suelen contarnos que la escritura "apareció" hace mucho tiempo, en una civilización muy lejana llamada Mesopotamia. Luego, "por alguna razón" se desarrolló en Egipto, Grecia y Roma hasta que en la Edad Media se expandió por toda Europa. El relato termina con la creación de la imprenta y de la visión de los libros como semilleros del pensamiento moderno. Después, todo se vuelve un recuento de episodios políticos.

Sobre este punto podemos hacernos las siguientes preguntas: ¿la imprenta fue un parteaguas realmente?, ¿cómo es que los libros sembraron el pensamiento moderno? y ¿hay alguna situación presente que puede compararse con el fenómeno de los libros impresos?
Imagen 1. Dos soportes de librería con escalas para libros de música (Crespi, 1720-1730)
Construido desde la reflexión docente, este artículo intenta responder a estas cuestiones mediante un argumento diseñado en dos premisas y una conclusión. La primera premisa es una interpretación novedosa sobre la imprenta, la segunda una comparación con un fenómeno presente, y la conclusión es una reflexión final sobre lo que representan las innovaciones tecnológicas.

\section{Piedra. Otra versión de la imprenta}

La imprenta de Gutenberg sí fue un parteaguas, pero hasta mucho tiempo después. En el siglo xv, tiempo en el que vivió Johannes Gutenberg, la imprenta tuvo poca influencia en la cultura europea debido al analfabetismo, al monopolio religioso y al incipiente mercado de bienes culturales.

El primer prototipo de imprenta de Gutenberg tuvo éxito en 1448, con una serie de calendarios y poemas. Posteriormente, se hicieron mejoras en el diseño y se imprimieron las famosas biblias de Gutenberg, objetos de colección altamente valiosos, tanto a nivel cultural como económico. Ésta es la versión más conocida del invento, aunque existe otro relato que expresa las dificultades que enfrentó el genio alemán. 
Gutenberg tuvo constantes conflictos con los inversionistas, por ejemplo, la construcción del prototipo tardó mucho más del tiempo esperado y los primeros impresos eran poco prometedores. Además, la iglesia católica mantenía el monopolio de la producción y el comercio de libros en Europa occidental, razón por la que las copias manuscritas que llegaron a venderse fuera de su consentimiento fueron clasificadas como clandestinas y actualmente pueden ser vendidas a precios altos para coleccionistas. A esto hay que sumar el problema estético: las primeras biblias impresas eran feas en comparación con las obras de arte de los escribanos religiosos, en su mayoría artesanos de letras, que hacían grabados bellísimos.

Sin lugar a duda, la imprenta representó un adelanto tecnológico para su tiempo, los libros artesanales tardaban años en elaborarse, eran costosos y solían terminar como un artículo de lujo para la nobleza. Por tanto, la imprenta mejoró la forma de producir libros, disminuyó el tiempo de fabricación y mejoró el diseño, respondiendo a las demandas de una burguesía emergente que deseaba educarse.

Éstos fueron los pilares del conocimiento escrito que afianzaron las bases del pensamiento moderno. Sin embargo, Gutenberg no vio nada de ello; al contrario, enfrentó varios juicios por fraude, fue señalado por herejía y afrontó el espionaje con el que, finalmente, robaron los secretos de su creación. La cuestión es: ¿cómo es que la imprenta pasó de ser un invento secreto en el sótano de Gutenberg a la gran innovación tecnológica que provocó una coyuntura cultural?

Imagen 2. William Caxton, impresor inglés, mostrando muestras de su impresión al rey Eduardo iv y su reina (1877).

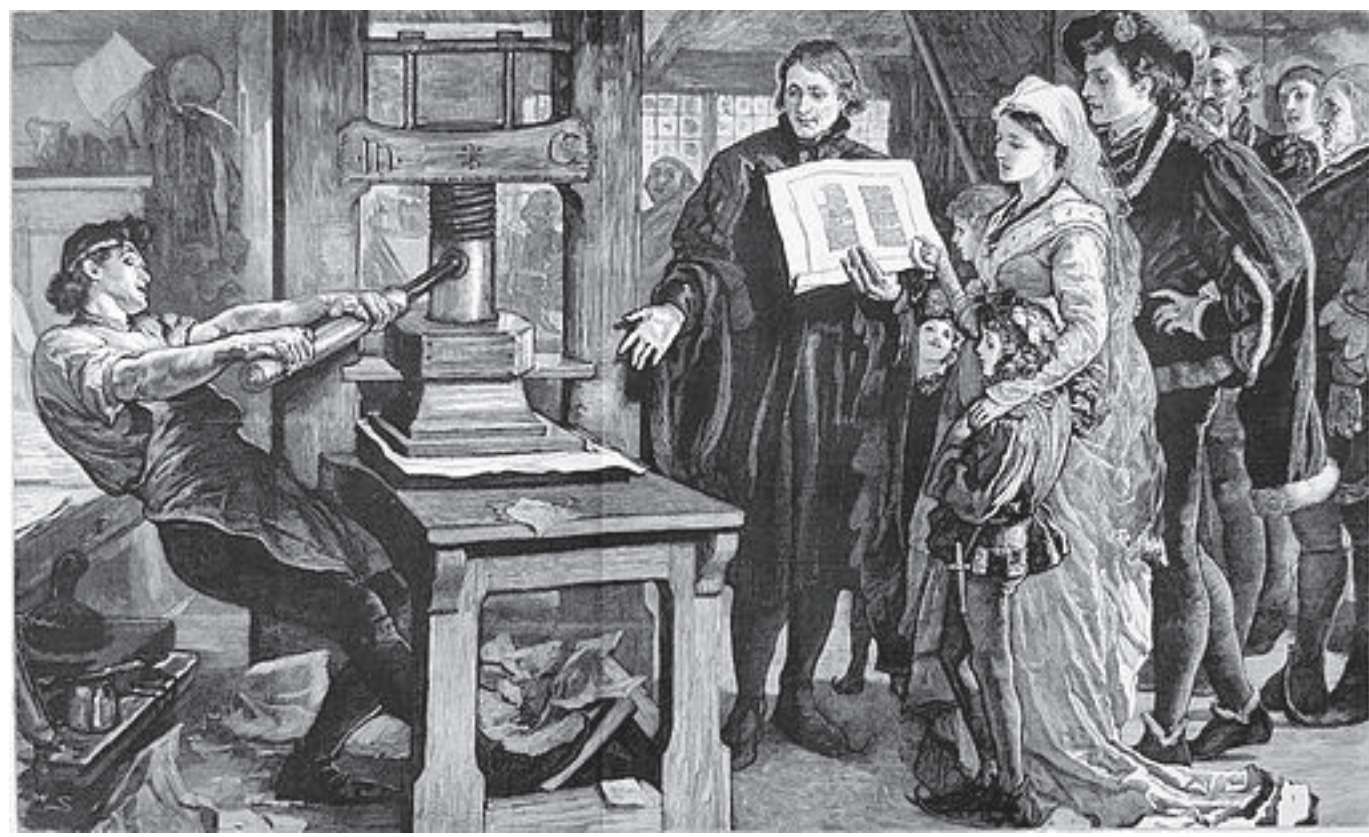

La respuesta está en su equipo de trabajo, actores sociales a los que se ha prestado poca atención debido a que en los relatos históricos, que todavía se 
Imagen 3. Audiolibros de Cuentos del Reino (Kingdomtales. com, 2019)

enseñan en las escuelas, aún pervive la idea del genio que lo cambia todo. Pero sucedió algo diferente: el cambio cultural causado por el libro impreso se debe a una construcción social distinta del conocimiento, a la suma de ideas, prácticas y saberes que muchas personas hacen en torno a un proyecto a lo largo del tiempo (Shapin, 2000).

En la obra El discípulo de Gutenberg, Alix Christie narra las dificultades técnicas, artísticas y económicas de la primera imprenta europea, señalando el papel transcendental que tuvo el equipo de trabajo del maestro Gutenberg: tres artesanos del gremio de orfebres de Maguncia y Peter Schöeffer, el joven escribano que llevó la escritura monástica medieval

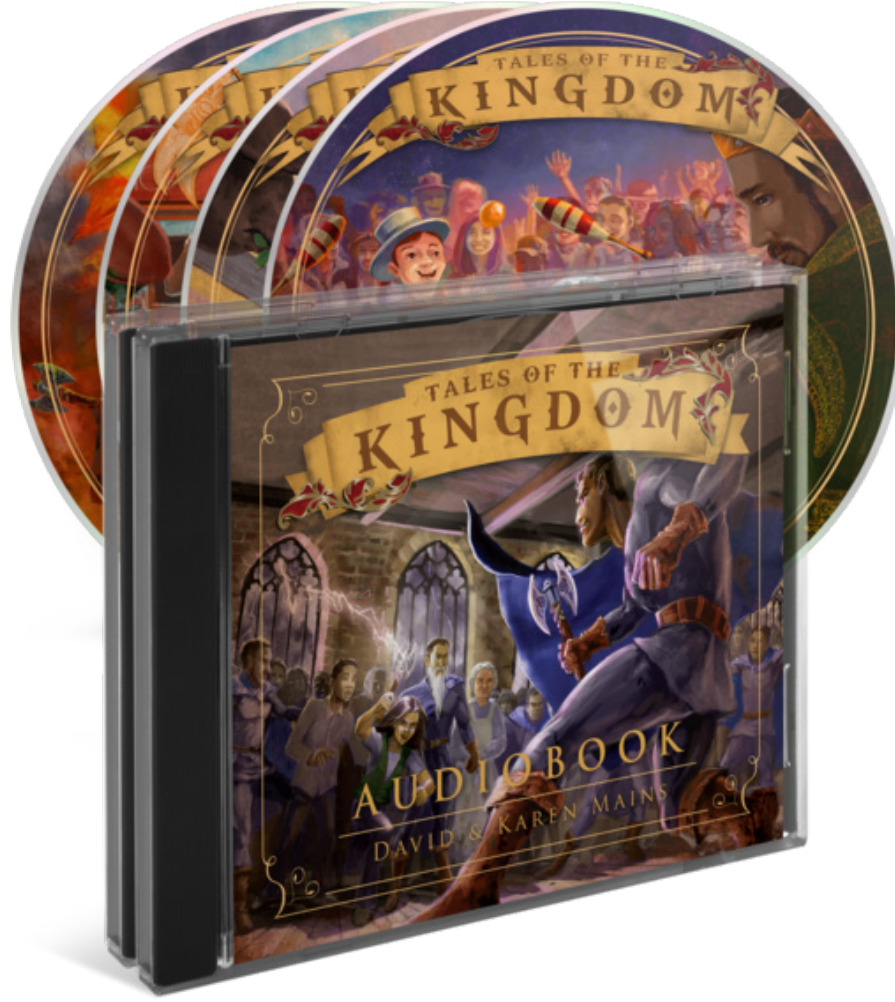
a los tipos (piezas metálicas que dan vida a las letras de impresión). De esta forma, la autora muestra cómo los problemas económicos de Gutenberg con los acreedores y las disputas entre artesanos terminaron por mermar su salud física y mental, tanto que no alcanzó a ver la grandeza de su invento.

Uno de los problemas técnicos que no resolvió, por ejemplo, fue la homogeneidad de los tipos, ya que el proceso de la elaboración se aceleró tanto que las hojas impresas mostraban un trabajo descuidado y con exceso de tinta, y tampoco obtuvo la venta esperada de sus primeros libros.

Poco después, Peter Schöeffer retomó y mejoró la imprenta, apoyándose en los vínculos que su familia tenía entre burgueses educados, con quienes emprendió la primera red comercial de libros. Desde entonces se conformaron más redes de lectores en el mundo occidental. De esta forma, el libro impreso liberó el conocimiento escrito del poder clerical y permitió su circulación mediante asociaciones comerciales, las cuales se expandieron por todo el mundo, llevando y trayendo ideas.

\section{Papel. Una coyuntura cultural en el siglo XXI}

Para conocer más sobre este proceso puede consultarse la Historia social del conocimiento: de Gutenberg a Diderot, de Peter Burke (2013, p. 320).
Para comprender mejor la aparición de los libros impresos podemos compararla con la de los audiolibros en aplicaciones digitales como Spotify. Los primeros audiolibros (ver imgen 3) surgieron con la revolución tecnológica en la música durante el siglo xx. No sólo se grabaron canciones y ritmos, sino también lecturas de libros en discos de vinil, casetes y discos compactos, aunque su impacto fue apenas perceptible en la industria editorial. Hasta la década de 1980 el libro impreso se siguió considerando el objeto cultural más importante en el mundo occidental, pues representaba la circulación del conocimiento en la era moderna. No obstante, una década más tarde ya nadie podía sostener esa premisa. 
Imagen 4. Spotify en un teléfono (Havarhen, 2014).
Dado que la música es una de las expresiones más importantes de la humanidad y forma parte de nuestra memoria auditiva, fungió como estrategia de enseñanza en el más grande esfuerzo de alfabetización emprendido por la cultura occidental, durante el siglo xx. En gran medida, gracias al invento de los reproductores móviles como los casetes de audio y video.

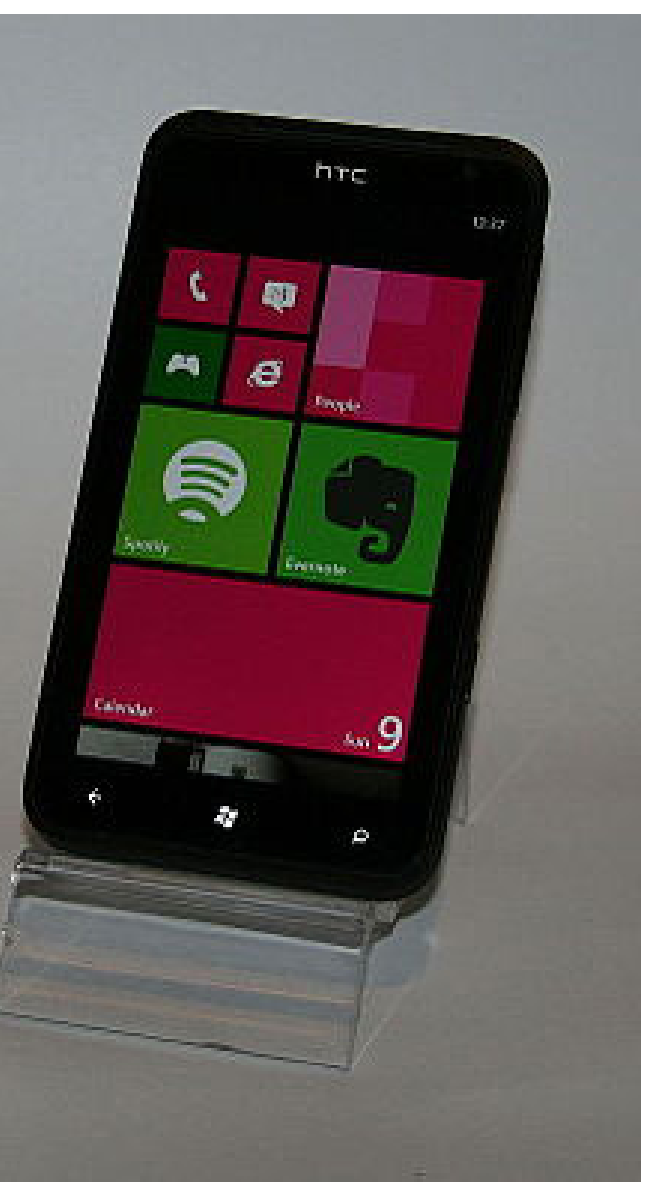

Pero los libros impresos siguieron siendo la base fundamental de la educación moderna y las novedades tecnológicas sólo se usaban como herramientas auxiliares, hasta que el internet cambió las reglas en la década de 1990. El veloz desarrollo de tecnologías digitales inició un proceso informático con sitios de interacción comunicativa y difusión del conocimiento que se multiplicaron de manera sorprendente.

La búsqueda de innovaciones para reproducir música en los teléfonos inteligentes dio pie a reproductores digitales como Spotify (ver imagen 4), que permite escuchar música a través de internet o, por una suscripción, descargar contenido. Spotify es una empresa sueca de streaming, fundada en 2006 por Daniel Ek y Martin Lorentzon, pero que se lanzó hasta el 7 de octubre de 2008 y que se ha distribuido en diversos países a través de los años. Sus listas de reproducción son casi infinitas y con la posibilidad de generar las propias. También se encuentran audiolibros y grabaciones llamadas pódcasts.

Estos formatos permiten la circulación del conocimiento de forma auditiva y, en comparación con los libros, su reproducción es más rápida, sencilla y gratuita. Los audiolibros y los pódcasts pueden compararse al invento de Gutenberg, ya que, tal como sucedió en el siglo Xv, producen dudas acerca el futuro de lo tradicionalmente aceptado. ¿Qué pasará con los libros impresos? ¿Podemos pensar la educación sin ellos?²

La nostalgia por los libros resulta hoy una constante que ha propiciado ciertos prejuicios hacia las nuevas tecnologías, como afirmar que los jóvenes mexicanos leen cada vez menos. Esto es falso por dos razones: el fundamento de la comunicación por internet, llamada popularmente red, necesita de una lectura continua; segundo, la base informática que da vida al universo digital es literalmente un manuscrito de códigos.

\section{Tijeras. Reflexión final}

La coyuntura cultural provocada por la imprenta fue un proceso multifactorial y el resultado de una construcción social de la diseminación conocimiento. Por esta razón, los libros tardaron siglos en convertirse en el objeto central de la 
Imagen 5. Micrositio Hubhopper Podcaster (Gautamranand, 2019).

2 Este artículo reflexivo nació de las discusiones de la asignatura Estudios Sociales y Económicos de México, en el Colegio de Bachilleres de la Ciudad de México. En gran medida está inspirado en la discusión sobre "Las batallas de la educación" que Fernando Escalante (2015, p.220) propone en la obra Historia mínima del neoliberalismo. Sobre el temor que generan los cambios científico-tecnológicos puede consultarse la obra llya Prigogine (1995, p. 146), El fin de las certidumbres. circulación del conocimiento. Algo similar pasa con las tecnologías digitales hoy en día. La fascinación que las rodea es tan grande como las dudas que propician.

De igual manera, se debe considerar que el universo digital ha creado una brecha tecnológica entre los lectores comunes y los alfabetos digitales, es decir, aquellas personas que saben manipular las nuevas tecnologías e, incluso, desarrollarlas. Ahora ya no se trata sólo de saber leer y escribir, sino de hacerlo usando las nuevas tecnologías. Personalmente, creo que ésta debe ser la preocupación principal en la educación del siglo xxI.

Sobre los impresos, puede afirmarse que los libros no dejarán de usarse y que su arraigo es tan profundo en nuestra cultura que se han desarrollado infinidad de formatos para trasladar su naturaleza al universo digital, dando como resultado los llamados libros digitales, eBook, ePub, PDF, etcétera. En efecto, la experiencia no es la misma, sin embargo, podríamos preguntarnos por qué debería ser así.

Finalmente, son dos las reflexiones con las quiero cerrar el artículo. Primera, los audiolibrosy los pódcasts son una nueva experiencia en la circulación del conocimiento (ver imagen 5) y aún desconocemos la coyuntura cultural que están causando, mientras no lo sepamos, los libros seguirán siendo el pilar de la educación moderna. Por último, el ingenio de Gutenberg se abrió paso frente al monopolio de la Iglesia y hoy en día parece que estamos en un umbral similar, donde las innovaciones tecnológicas nos invitan a ser protagonistas de la construcción del conocimiento. ¿Podemos preguntarnos, entonces, si deberíamos cambiar la nostalgia de los libros impresos por la oportunidad que nos brinda el porvenir digital en la educación?²
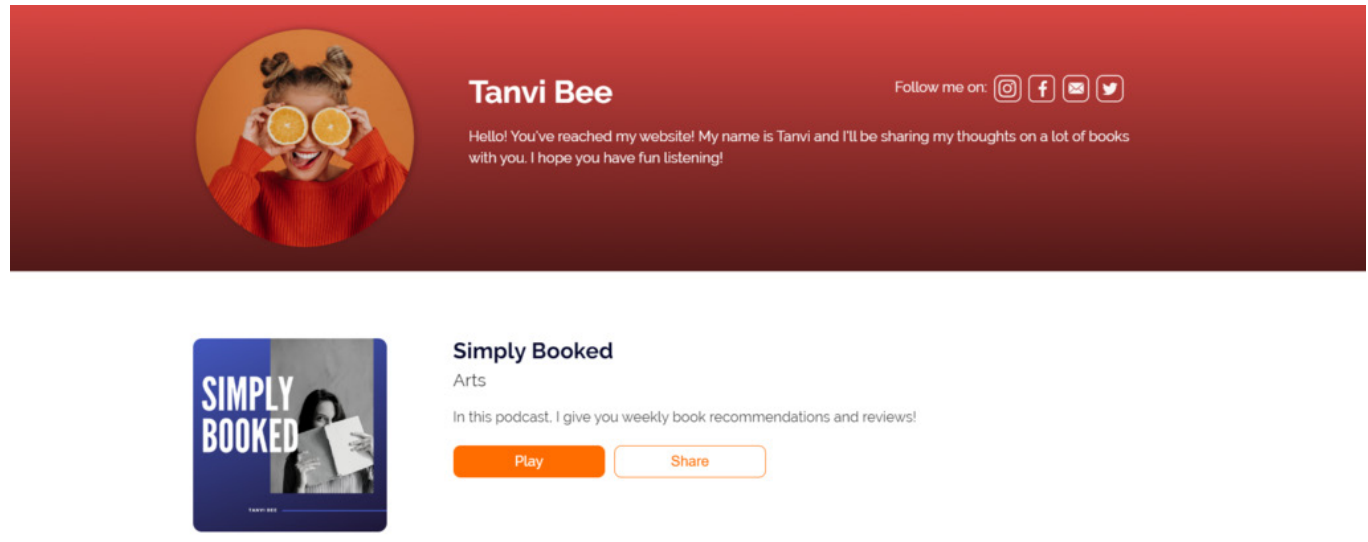

Simply Booked

Arts

In this podcast. I give you weekly book recommendations and reviews

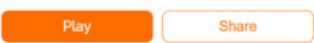

Latest Episodes
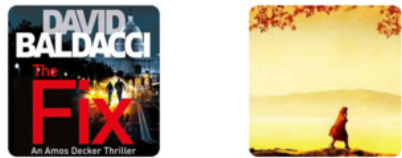


\section{Referencias}

* Burke, Peter. (2013). Historia social del conocimiento: de Gutenberg a Diderot. Planeta.

* The Caxton Celebration - William Caxton showing specimens of his printing to King Edward Iv and his Queen.jpg. (1877, 30 de junio). The Graphic. https://commons. wikimedia.org/wiki/File:The Caxton Celebration - William Caxton showing specimens of his printing to King Edward IV and his Queen.jpg.

* Crespi, Giuseppe. (1720-1730) Due_sportelli_di_libreria_con_scaffali_di_libri_di_ musica.jpg. https://commons.wikimedia.org/wiki/File:Due sportelli di libreria con scaffali di libri di musica.jpg.

* Darnton, Robert. (1987). La gran matanza de gatos y otros episodios en la historia de la cultura francesa. Fondo de Cultura Económica.

* Escalante, Fernando. (2015). Historia mínima de El Neoliberalismo. El Colegio de México.

* Foucault, Michael. (1968). Las palabras y las cosas: una arqueología de las ciencias humanas. Siglo XXI.

* Gautamranand. (2019). Hubhopper microsite.png. https://commons.wikimedia.org/ wiki/File:Hubhopper microsite.png.

* Havarhen (2014, 9 de febrero). A нтc Titan Windows Phone device. https://commons. wikimedia.org/wiki/File:HTC Titan.jpeg.

Kingdomtales.com (2019). Tales of the Kingdom Audiobook. https://commons. wikimedia.org/wiki/File:Tales of the Kingdom Audiobook.png.

* Latour, Bruno. (2001). La esperanza de Pandora: ensayos sobre la realidad de los estudios de la ciencia. Gedisa.

* Prigogine, llya. (1995). El fin de las certidumbres. Andrés Bello.

* Shapin, Steven. (2000). La revolución científica. Una interpretación alternativa. Paidós.

* Tanck, Dorothy. (2010). Historia mínima de la educación en México. El Colegio de México.

\section{Cómo citar el artículo}

* González, Leonardo A. (2020) ¿Piedra, papel o tijeras? La imprenta y Spotify como innovaciones tecnológicas. Revista Digital Universitaria (RDU) Vol. 21, núm. 3 mayojunio. Dol: http://doi.org/10.22201/codeic.16076079e.2020.v21n3.a8.

Recepción: 13/06/2018. Aceptación: 22/10/2019. 